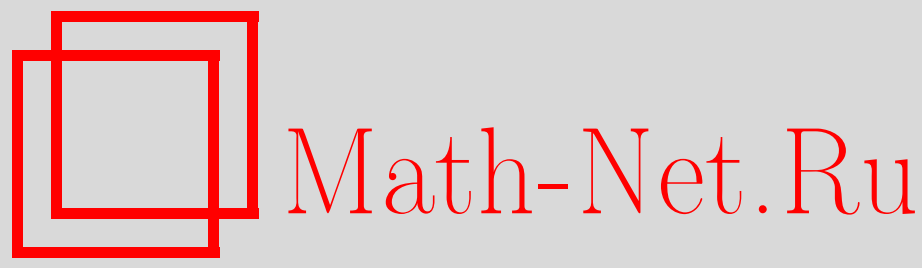

А. В. Латышев, А. А. Юшканов, Продольная диэлектрическая проницаемость квантовой вырожденной столкновительной плазмы, ТМФ, 2011, том 169, номер 3, 431-443

DOI: https://doi.org/10.4213/tmf6739

Использование Общероссийского математического портала Math-Net.Ru подразумевает, что вы прочитали и согласны с пользовательским соглашением http://www.mathnet.ru/rus/agreement

Параметры загрузки:

IP : 34.229 .108 .108

26 апреля 2023 г., $11: 44: 11$

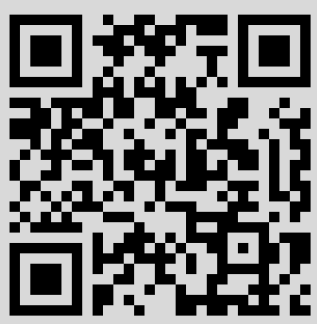




\title{
ПРОДОЛЬНАЯ ДИЭЛЕКТРИЧЕСКАЯ ПРОНИЦАЕМОСТЬ КВАНТОВОЙ ВЫРОЖДЕННОЙ СТОЛКНОВИТЕЛЬНОЙ ПЛАЗМЫ
}

\begin{abstract}
Найдена диэлектрическая проницаемость вырожденного электронного газа для столкновительной плазмы. Используется кинетическое уравнения Вигнера-Власова-Больцмана с интегралом столкновений в релаксационной форме в координатном пространстве. Исследованы коновские особенности диэлектрической проницаемости и выявлено их размывание в бесстолкновительной плазме.
\end{abstract}

Ключевые слова: вырожденный электронный газ, диэлектрическая проницаемость, интеграл столкновений, функция Линдхардта, коновские особенности.

\section{1. ВВЕДЕНИЕ}

В настоящей работе выводятся формулы для проводимости и диэлектрической проницаемости квантовой электронной плазмы.

Диэлектрическая проницаемость является одной из важнейших характеристик плазмы. Эта величина необходима для описания процесса распространения и затухания плазменных колебаний, скин-эффекта, механизма проникновения электромагнитных волн в плазму [1]-[3] и для анализа других проблем в физике плазмы [4].

Диэлектрическая проницаемость в бесстолкновительной квантовой газовой плазме изучалась многими авторами (см., например, работы [5]-[10]). В работе [8], где исследовался одномерный случай, отмечалась важность вывода диэлектрической проницаемости с использованием квантового кинетического уравнения с интегралом столкновений в форме модели Бхатнагара-Гросса-Крука [11] (БГК-модели).

Настоящая работа посвящена выполнению этой задачи. Для ее решения мы применяем квантовое кинетическое уравнение Больцмана-Вигнера-Власова (БВВ-уравнение) с интегралом столкновений в форме $\tau$-модели, называемым БГК-интегралом столкновений.

БВВ-уравнение записывается относительно функции Вигнера, которая является аналогом функции распределения электронов для квантовой плазмы [12], [13].

* Московский государственный областной университет, Москва, Россия. E-mail: avlatyshev@mail.ru,yushkanov@inbox.ru 
Наиболее распространенным методом исследования квантовой плазмы является метод Хартри-Фока или эквивалентный ему метод приближения случайных фаз [14], [15].

В работе [16] этим методом получено выражение для диэлектрической проницаемости квантовой плазмы в $\tau$-приближении, однако в работе [17] показано, что полученное выражение некорректно, так как не переходит в классическое выражение при условии пренебрежения квантовыми поправками. В работе [17] было предложено эмпирически подправленное выражение для диэлектрической проницаемости квантовой плазмы, свободное от указанного недостатка, с помощью которого авторы исследовали квантовые поправки к оптическим свойствам металла [18], [19].

Диэлектрическая проницаемость квантовой плазмы широко используется также для изучения экранировки электрического поля и возникающих при этом фриделевских осцилляций (см., например, работы [20]-[22]). В работе [23] изучена экранировка кулоновского поля в намагниченном электронном газе квантового цилиндра.

В теории квантовой плазмы существуют две принципиально различные возможности построения релаксационного кинетического уравнения в $\tau$-приближении: в пространстве импульсов (в пространстве фурье-образов функции распределения) и в пространстве координат. На основании кинетического релаксационного уравнения в пространстве импульсов Мермином [24] в 1970 г. впервые был проведен последовательный вывод диэлектрической проницаемости для квантовой столкновительной плазмы. Отметим, что важность учета влияния столкновений на статическую продольную проницаемость квантовой плазмы при рассмотрении свойств сплавов подчеркивалась в работе [25].

Диэлектрические свойства квантовой столкновительной плазмы до сих пор остаются мало исследованной областью. По сути дела, единственной работой, в которой предпринята попытка корректно описать влияние столкновений на диэлектрические свойства квантовой плазмы, остается известная работа Мермина [24]. Однако даже в классическом случае описание кинетических процессов в газе и плазме существенно зависит от выбора вида интеграла столкновений. При этом широко используются модельные интегралы столкновений БГК с постоянной и переменной частотой столкновений, эллипсоидально-статистический интеграл столкновений, интеграл столкновений Шахова и т. д. Это отнюдь не является прихотью, так как для разных ситуаций наиболее адекватны различные интегралы столкновений. В квантовом случае ситуация еще более усложняется. Даже простейший релаксационный интеграл столкновений может иметь различные обобщения на квантовый случай. Возникает естественный вопрос: какие свойства диэлектрической проницаемости Мермина являются общими свойствами квантового описания плазмы, а какие - следстием выбора конкретного интеграла столкновений? Единственный способ выяснить это - исследовать квантовую диэлектрическую проницаемость с помощью другого, чем у Мермина, интеграла столкновений. Нами был выбран интеграл столкновений БГК. При этом наиболее удобно было вести рассмотрение с использованием функции Вигнера.

В настоящей работе выведено выражение для продольной диэлектрической проницаемости с использованием релаксационного уравнения в пространстве координат. Если в полученном выражении постоянную Планка устремить к нулю $(\hbar \rightarrow 0)$, 
получим в точности классическое выражение диэлектрической проницаемости невырожденной плазмы. Исследованы различные предельные случаи диэлектрической проницаемости и проведено сравнение с результатом Мермина.

\section{2. РЕШЕНИЕ КИНЕТИЧЕСКОГО УРАВНЕНИЯ}

Рассмотрим кинетическое БВВ-уравнение [26]:

$$
\frac{\partial f}{\partial t}+\mathbf{v} \frac{\partial f}{\partial \mathbf{r}}=-\frac{i e}{\hbar} W[f]+B[f, f] .
$$

Здесь $e$ - заряд электрона, $W[f]$ - функционал Вигнера-Власова от потенциала,

$$
\begin{aligned}
W[f]= & \frac{1}{(2 \pi)^{3}} \int\left[U\left(\mathbf{r}+\frac{\hbar \mathbf{b}}{2}, t\right)-U\left(\mathbf{r}-\frac{\hbar \mathbf{b}}{2}, t\right)\right] \times \\
& \times f\left(\mathbf{r}, \mathbf{p}^{\prime}, t\right) e^{i \mathbf{b}\left(\mathbf{p}^{\prime}-\mathbf{p}\right)} d^{3} b d^{3} p^{\prime}
\end{aligned}
$$

$\mathbf{b}=\left\{b_{x}, b_{y}, b_{z}\right\}-$ вектор, $f=f(\mathbf{r}, \mathbf{p}, t)-$ функция Вигнера для электронов, $\hbar-$ постоянная Планка, $U(\mathbf{r}, t)$ - скалярный потенциал электрического поля, $B[f, f]-$ интеграл столкновений.

Интеграл столкновений в общем случае для квантовой плазмы может иметь весьма сложный вид. В частности, он может быть и не локальным по координатам. Предельный случай такой квантовой нелокальности рассмотрен в работе [24]. В настоящей работе рассмотрен случай, когда интеграл столкновений можно представить в локальной форме. Конкретно, мы рассмотрим представление интеграла столкновений в форме стандарного модельного БГК-интеграла столкновений [11] (см. также работу [27], где проведено исследование этой модели). Тогда предыдущее уравнение перепишется в виде

$$
\frac{\partial f}{\partial t}+\mathbf{v} \frac{\partial f}{\partial \mathbf{r}}=-\frac{i e}{\hbar} W[f]+\nu\left[f_{\mathrm{eq}}(\mathbf{r}, \mathbf{p}, t)-f(\mathbf{r}, \mathbf{p}, t)\right]
$$

Здесь $\nu$ - эффективная частота рассеяния электронов (в частности, на примесях), $f_{\text {eq }}$ - равновесная функция Ферми распределения электронов. В дальнейшем мы будем рассматривать достаточно низкие температуры, чтобы можно было считать электронный газ вырожденным. Тогда равновесная функция распределения выражается через функцию Хевисайда: $f_{\text {eq }}=\Theta\left(\mathcal{E}_{\text {eq }}(\mathbf{r}, t)-\mathcal{E}\right)$, где функция $\Theta(x)-$ функция Хевисайда, $\Theta(x)=1, x>0 ; \Theta(x)=0, x<0 ; \mathcal{E}=p^{2} /(2 m)$ - кинетическая энергия электронов; $\mathcal{E}_{\mathrm{F}}(\mathbf{r}, t)=p_{\mathrm{F}}^{2}(\mathbf{r}, t) / 2 m$ - возмущенная энергия Ферми электрона; $\mathbf{p}=m \mathbf{v}-$ импульс электрона; $\mathbf{p}_{\mathrm{F}}-$ импульс электрона на поверхности Ферми, которую мы считаем сферической.

Используемое кинетическое уравнение (2.2) учитывает релаксацию неравновесного состояния к локальному термодинамическому равновесию.

Будем считать, что функция распределения электронов зависит от одной пространственной координаты $x$, времени $t$ и импульса $\mathbf{p}$, а скалярный потенциал электрического поля зависит от одной пространственной координаты $x$ и времени $t$ : $U(x, t)=U_{0} e^{i(k x-\omega t)}$.

Линеаризацию уравнений (2.1) и (2.2) проведем относительно абсолютного фермиана $f_{\mathrm{F}}(p)=\Theta\left(\mathcal{E}_{\mathrm{F}}-\mathcal{E}\right)$, где $\mathcal{E}_{\mathrm{F}}-$ энергия электрона на поверхности Ферми, $\mathcal{E}_{\mathrm{F}}=$ $p_{\mathrm{F}}^{2} /(2 m)$. 
Линеаризация функции Вигнера для электронов и равновесной функции распределения приводит к равенствам

$$
\begin{aligned}
f & =f_{\mathrm{F}}(p)+U_{0} e^{i(k x-\omega t)} f_{1}(\mathbf{p}), \\
f_{\mathrm{eq}} & =f_{\mathrm{F}}(p)+\left[\mathcal{E}_{\mathrm{F}}(x, t)-\mathcal{E}_{\mathrm{F}}\right] \delta\left(\mathcal{E}_{\mathrm{F}}-\mathcal{E}\right),
\end{aligned}
$$

где $f_{1}(\mathbf{p})$ - новая неизвестная функция, $\delta(x)$ - дельта-функция Дирака.

Величина $\mathcal{E}_{\mathrm{F}}(x, t)-\mathcal{E}_{\mathrm{F}}$ описывает локальное изменение энергии Ферми электронного газа, обусловленное изменением его плотности. Наличие этого слагаемого в интеграле столкновений обеспечивает выполнение закона сохранения числа электронов.

В линейном приближении функционал Вигнера преобразуется к виду

$$
W[f]=U(x, t)\left[\Theta_{+}(\mathbf{v})-\Theta_{-}(\mathbf{v})\right],
$$

где

$$
\Theta_{ \pm}(\mathbf{v})=\Theta\left[v_{\mathrm{F}}^{2}-\left(v_{x} \mp \frac{\hbar k}{2 m}\right)^{2}-v_{y}^{2}-v_{z}^{2}\right] .
$$

Подставив выражения (2.3) и (2.4) в уравнение (2.2), получаем:

$$
f_{1}(\mathbf{p})\left(\nu-i \omega+i k v_{x}\right) U(x, t)=-\frac{i e}{\hbar} W[f]+\nu \delta\left(\mathcal{E}_{\mathrm{F}}-\mathcal{E}\right)\left(\mathcal{E}_{\mathrm{F}}(x, t)-\mathcal{E}_{\mathrm{F}}\right) .
$$

Величину $\mathcal{E}_{\mathrm{F}}(x, t)-\mathcal{E}_{\mathrm{F}}$ найдем из закона сохранения числа частиц. В результате получаем

$$
\mathcal{E}_{\mathrm{F}}(x, t)-\mathcal{E}_{\mathrm{F}}=U(x, t) \frac{m A}{4 \pi v_{\mathrm{F}}},
$$

где

$$
A=\int f_{1}(\mathbf{p}) d^{3} v
$$

Тогда из уравнений (2.5)-(2.7) следует:

$$
f_{1}(\mathbf{p})=-\frac{i e}{\hbar} \frac{\Theta_{+}(\mathbf{v})-\Theta_{-}(\mathbf{v})}{\nu+i\left(k v_{x}-\omega\right)}+\frac{A \nu}{4 \pi v_{\mathrm{F}}^{2}} \frac{\delta\left(v_{\mathrm{F}}-v\right)}{\nu+i\left(k v_{x}-\omega\right)} .
$$

\section{3. ПРОДОЛЬНАЯ ПРОНИЦАЕМОСТЬ И ПРОВОДИМОСТЬ}

Подставляя выражение (2.8) в соотношение (2.7), получаем уравнение

$$
A=-\frac{i e}{\hbar} \int \frac{\Theta_{+}(\mathbf{v})-\Theta_{-}(\mathbf{v})}{\nu+i\left(k v_{x}-\omega\right)} d^{3} v+\frac{A \nu}{4 \pi v_{\mathrm{F}}^{2}} \int \frac{\delta\left(v_{\mathrm{F}}-v\right) d^{3} v}{\nu+i\left(k v_{x}-\omega\right)},
$$

из которого находим

$$
A=-\frac{i e}{\hbar} \frac{J(\omega, k, \nu)}{1-g_{0}(\omega, k, \nu)}
$$

где введены обозначения

$$
\begin{aligned}
g_{0}(\omega, k, \nu) & =\frac{i \nu}{2 k v_{\mathrm{F}}} \ln \frac{\omega+i \nu+k v_{\mathrm{F}}}{\omega+i \nu-k v_{\mathrm{F}}}, \\
J(\omega, k, \nu) & =\int \frac{\Theta_{+}(\mathbf{v})-\Theta_{-}(\mathbf{v})}{\nu+i\left(k v_{x}-\omega\right)} d^{3} v .
\end{aligned}
$$


При выводе формулы (3.2) было использовано вычисление интеграла в сферических координатах:

$$
\int \frac{\delta\left(v_{\mathrm{F}}-v\right) d^{3} v}{\nu+i\left(k v_{x}-\omega\right)}=2 \pi v_{\mathrm{F}}^{2} \int_{-1}^{1} \frac{d \mu}{\nu+i\left(k v_{\mathrm{F}} \mu-\omega\right)}=4 \pi v_{\mathrm{F}}^{2} g_{0}(\omega, k, \nu) .
$$

Воспользовавшись далее определением функций $\Theta_{ \pm}(\mathbf{v})$ для интеграла (3.3), получаем $J=J^{+}-J^{-}$, где

$$
J^{ \pm}(\omega, k, \nu)=\int_{S_{ \pm}^{3}} \frac{d v_{x} d v_{y} d v_{z}}{\nu+i\left(k v_{x}-\omega\right)}
$$

Здесь $S_{ \pm}^{3}$ - шар с центром в точке $(\mp \hbar k /(2 m), 0,0)$, радиусом которого является скорость электрона на поверхности Ферми:

$$
S_{ \pm}^{3}: \quad\left(v_{x} \pm \frac{\hbar k}{2 m}\right)^{2}+v_{y}^{2}+v_{z}^{2} \leqslant v_{\mathrm{F}}^{2}
$$

После очевидной замены переменной $v_{x} \pm \hbar k /(2 m) \rightarrow v_{x}$ получаем

$$
J^{ \pm}=\int_{S^{3}(0)} \frac{d v_{x} d v_{y} d v_{z}}{\nu+i k\left(v_{x} \pm \hbar k /(2 m)\right)-i \omega}
$$

где $S^{3}(0)$ - шар Ферми с центром в начале координат: $v_{x}^{2}+v_{y}^{2}+v_{z}^{2} \leqslant v_{\mathrm{F}}^{2}$.

Шар Ферми $S^{3}(0)$ представим в виде объединения:

$$
S^{3}(0)=\bigcup_{v_{x}=-v_{\mathrm{F}}}^{v_{x}=v_{\mathrm{F}}} S_{v_{\mathrm{F}}^{2}-v_{x}^{2}}^{2}(0,0)
$$

Здесь $S_{v_{\mathrm{F}}^{2}-v_{x}^{2}}^{2}(0,0)$ есть круг вида $v_{y}^{2}+v_{z}^{2}<v_{\mathrm{F}}^{2}-v_{x}^{2}$. Теперь интегралы $J^{ \pm}$вычислим как повторные:

$$
\begin{aligned}
J^{ \pm} & =\pi \int_{-v_{\mathrm{F}}}^{v_{\mathrm{F}}} \frac{\left(v_{\mathrm{F}}^{2}-v_{x}^{2}\right) d v_{x}}{\nu+i k\left(v_{x} \pm \hbar k /(2 m)\right)-i \omega}= \\
& =\frac{2 i \pi \nu v_{\mathrm{F}}}{k^{2}}\left(\omega_{\mp}+i \nu\right)-\frac{i \pi \nu}{k^{3}}\left[\left(\omega_{\mp}+i \nu\right)^{2}-k^{2} v_{\mathrm{F}}^{2}\right] \ln \frac{\omega_{\mp}+i \nu+k v_{\mathrm{F}}}{\omega_{\mp}+i \nu-k v_{\mathrm{F}}}
\end{aligned}
$$

где

$$
\omega_{ \pm}=\omega \pm \frac{\hbar k^{2}}{2 m}
$$

Разность интегралов $J^{+}$и $J^{-}$равна

$$
\begin{aligned}
J= & -\frac{2 i \pi \nu v_{\mathrm{F}} \hbar}{m}+\frac{i \pi \nu}{k^{3}}\left[\left(\omega_{+}+i \nu\right)^{2}-k^{2} v_{\mathrm{F}}^{2}\right] \ln \frac{\omega_{+}+i \nu+k v_{\mathrm{F}}}{\omega_{+}+i \nu-k v_{\mathrm{F}}}- \\
& -\frac{i \pi \nu}{k^{3}}\left[\left(\omega_{-}+i \nu\right)^{2}-k^{2} v_{\mathrm{F}}^{2}\right] \ln \frac{\omega_{-}+i \nu+k v_{\mathrm{F}}}{\omega_{-}+i \nu-k v_{\mathrm{F}}}
\end{aligned}
$$

или

$$
J=-\frac{2 i \pi \nu v_{\mathrm{F}} \hbar}{m}\left[1-g\left(\omega_{+}, k, \nu\right)+g\left(\omega_{-}, k, \nu\right)\right]
$$


где

$$
g\left(\omega_{ \pm}, k, \nu\right)=\frac{m\left[\left(\omega_{ \pm}+i \nu\right)^{2}-k^{2} v_{\mathrm{F}}^{2}\right]}{2 \hbar k^{3} v_{\mathrm{F}}} \ln \frac{\omega_{ \pm}+i \nu+k v_{\mathrm{F}}}{\omega_{ \pm}+i \nu-k v_{\mathrm{F}}} .
$$

Следовательно, согласно формулам (2.8) и (3.1), построена и функция $f_{1}(\mathbf{p})$ :

$$
f_{1}(\mathbf{p})=-\frac{i e}{\hbar}\left[\frac{\Theta_{+}(\mathbf{v})-\Theta_{-}(\mathbf{v})}{\nu+i\left(k v_{x}-\omega\right)}+\frac{J}{4 \pi v_{\mathrm{F}}^{2}\left(1-g_{0}\right)} \frac{\delta\left(v_{\mathrm{F}}-v\right)}{\nu+i\left(k v_{x}-\omega\right)}\right],
$$

где $J$ определяется равенствами (3.5) и (3.6), а $g_{0}$ - равенством (3.2).

Рассмотрим связь электрического поля и потенциала $\mathbf{E}=-\operatorname{grad} U$ и уравнение непрерывности для плотности тока и заряда $\partial \rho / \partial t+\partial j_{x} / \partial x=0$. Здесь согласно определению диэлектрической проводимости $j_{x}=\sigma_{1} E_{x}=-\sigma_{1} i k U(x, t)$. Следовательно, $\partial j_{x} / \partial x=\sigma_{1} k^{2} U(x, t)$.

Учитывая, что для плотности заряда выполняется очевидное равенство

$$
\rho=e \int f d \Omega_{\mathrm{F}}=e \int\left[f_{0}(\mathcal{E})+U(x, t) f_{1}\right] d \Omega_{\mathrm{F}},
$$

получаем

$$
\frac{\partial \rho}{\partial t}=-i \omega e U(x, t) \int f_{1} d \Omega_{\mathrm{F}}
$$

Воспользовавшись теперь уравнением непрерывности, находим общую формулу для вычисления продольной проводимости:

$$
\sigma_{1}=\frac{i e \omega}{k^{2}} \int f_{1} d \Omega_{\mathrm{F}}=\frac{2 i e \omega m^{3}}{(2 \pi \hbar)^{3} k^{2}} \int f_{1} d^{3} v
$$

Подставляя функцию (3.7) в формулу (3.8) и пользуясь выражениями (3.2) и (3.3), получаем

$$
\sigma_{1}=\frac{i \pi e^{2} \omega m^{3} v_{\mathrm{F}}}{2 \pi^{3} \hbar^{3} m k^{2}} \frac{J(\omega, k, \nu)}{1-g_{0}(\omega, k, \nu)},
$$

или, с учетом формул для числовой плотности электронов $3 \pi^{2} N=\left(m v_{\mathrm{F}} / \hbar\right)^{3}$ и классической проводимости $\sigma_{0}=e^{2} N / m \nu$,

$$
\sigma_{1}=-\frac{3 i \sigma_{0} \omega \nu}{2\left(k v_{\mathrm{F}}\right)^{2}} \frac{1-g\left(\omega_{+}, k, \nu\right)+g\left(\omega_{-}, k, \nu\right)}{1-g_{0}(\omega, k, \nu)} .
$$

Используя определение диэлектрической проницаемости $\varepsilon_{1}=1+4 \pi i \sigma_{1} / \omega$, с помощью формулы (3.9) можно получить выражение для продольной диэлектрической проницаемости плазмы:

$$
\varepsilon_{1}(\omega, k, \nu)=1+\frac{3 \omega_{\mathrm{p}}^{2}}{2 k^{2} v_{\mathrm{F}}^{2}} \frac{1-g\left(\omega_{+}, k, \nu\right)+g\left(\omega_{-}, k, \nu\right)}{1-g_{0}(\omega, k, \nu)},
$$

где $\omega_{\mathrm{p}}$ - плазменная частота, $\omega_{\mathrm{p}}^{2}=4 \pi e^{2} N / m$.

Введем безразмерные параметры

$$
z=x+i y, \quad x=\frac{\omega}{k v_{\mathrm{F}}}, \quad y=\frac{\nu}{k v_{\mathrm{F}}}, \quad x_{\mathrm{p}}=\frac{\omega_{\mathrm{p}}}{k v_{\mathrm{F}}}, \quad q=\frac{k}{k_{\mathrm{F}}},
$$

где $k_{\mathrm{F}}=m v_{\mathrm{F}} / \hbar-$ волновое число Ферми. 


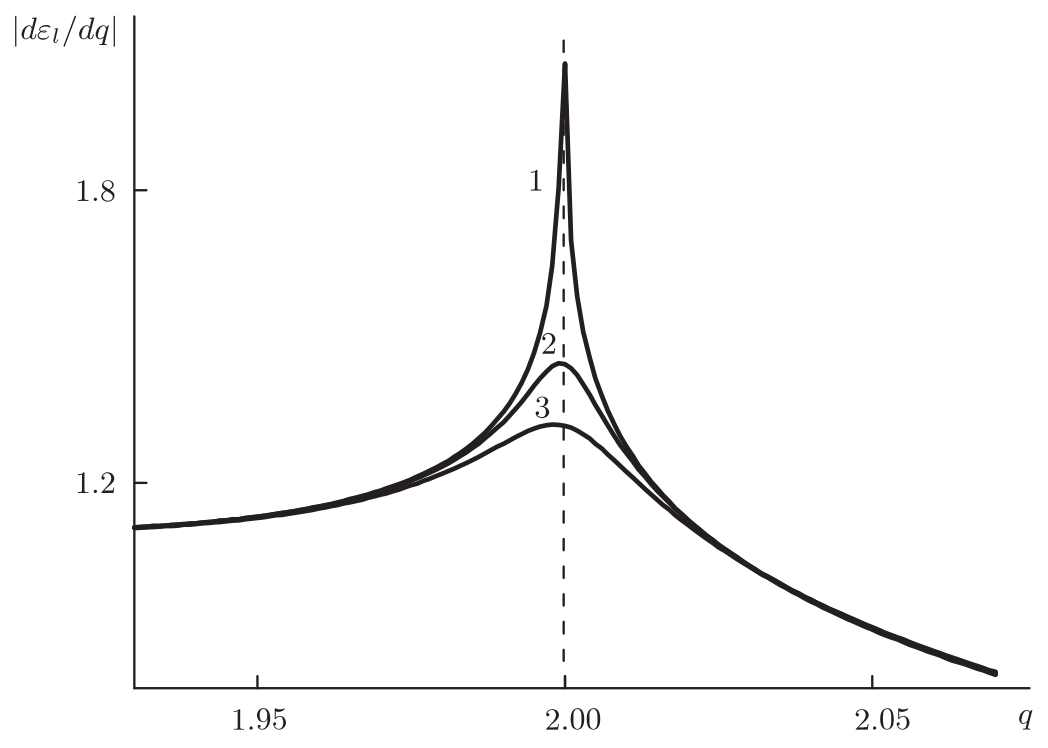

Рис. 1. Коновская особенность при $x_{\mathrm{p}}=1, x=0$.

В параметрах (3.11) безразмерную диэлектрическую проницаемость можно записать в виде

$$
\varepsilon_{1}(x, y, q)=1+\frac{3}{2} x_{\mathrm{p}}^{2} \frac{1-g(z,+q)+g(z,-q)}{1-g_{0}(x, y)},
$$

где

$$
g_{0}=\frac{i y}{2} \ln \frac{x+i y+1}{x+i y-1}, \quad g(z, \pm q)=\frac{(z \pm q / 2)^{2}-1}{2 q} \ln \frac{z \pm q / 2+1}{z \pm q / 2-1} .
$$

Пусть $\nu=0$, т. е. плазма является бесстолкновительной. Тогда из выражения (3.10) вытекает следующая классическая формула для диэлектрической проницаемости:

$$
\varepsilon_{\mathrm{l}}(\omega, k)=1+\frac{3 \omega_{\mathrm{p}}^{2}}{2 k^{2} v_{\mathrm{F}}}\left[1-g\left(\omega_{-}, k\right)+g\left(\omega_{+}, k\right)\right]
$$

где

$$
g\left(\omega_{ \pm}, k\right)=\frac{m\left(\omega_{ \pm}^{2}-k^{2} v_{\mathrm{F}}^{2}\right)}{2 \hbar k^{3} v_{\mathrm{F}}} \ln \frac{\omega_{ \pm}+k v_{\mathrm{F}}}{\omega_{ \pm}-k v_{\mathrm{F}}} .
$$

В литературе эта формула называется (см., например, работы [20]-[22]) диэлектрической функцией Линдхарда [16]. Она выводится методом приближения случайных фаз.

В безразмерных переменных (3.11) диэлектрическая функция Линдхарда имеет вид

$$
\varepsilon_{1}(x, q)=1+\frac{3}{2} x_{\mathrm{p}}^{2}[1-g(x,+q)+g(x,-q)]
$$

где

$$
g(x, \pm q)=\frac{(x \pm q / 2)^{2}-1}{2 q} \ln \frac{x \pm q / 2+1}{x \pm q / 2-1}
$$




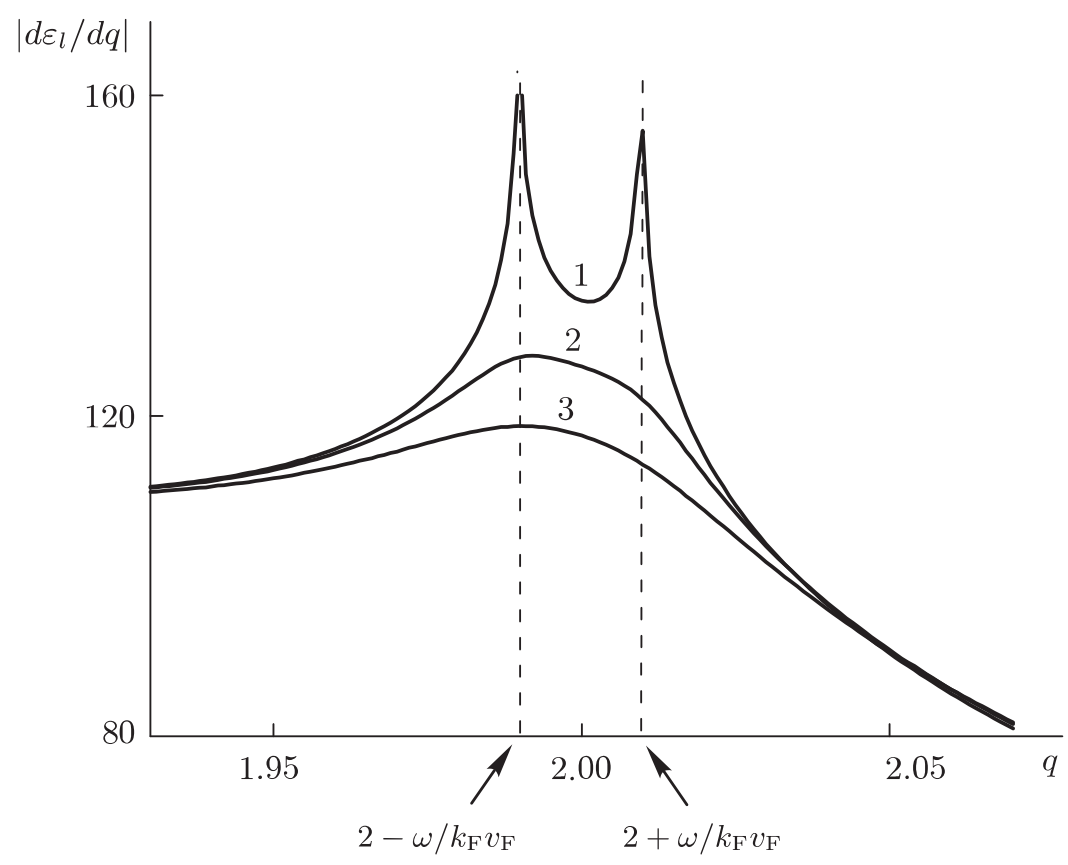

Рис. 2. Коновская особенность при $x_{\mathrm{p}}=10, x=0$.

На рис. 1 изображена коновская особенность в случае $x_{\mathrm{p}}=1, x=0$. Кривые $1,2,3$ построены для значений безразмерной частоты столкновений $y=0,0.005,0.01$ соответственно.

На рис. 2 и рис. 3 показаны коновские особенности в случае $x_{\mathrm{p}}=10, x=0$; кривые $1,2,3$ построены для значений безразмерной частоты столкновений $y=0,0.05,0.01$ соответственно.

Перейдя к новым безразмерным переменным

$$
z=x+i y, \quad x=\frac{\omega}{k_{\mathrm{F}} v_{\mathrm{F}}}, \quad y=\frac{\nu}{k_{\mathrm{F}} v_{\mathrm{F}}}, \quad x_{\mathrm{p}}=\frac{\omega_{\mathrm{p}}}{k_{\mathrm{F}} v_{\mathrm{F}}}, \quad q=\frac{k}{k_{\mathrm{F}}},
$$

получаем следующую формулу для продольной диэлектрической проницаемости:

$$
\varepsilon_{1}(x, y, q)=1+\frac{3 x_{\mathrm{p}}^{2}}{2 q^{2}} \frac{1-g_{+}(z, q)+g_{-}(z, q)}{1-g_{0}(x, y, q)},
$$

где

$$
g_{0}=\frac{i y}{2 q} \ln \frac{x+i y+q}{x+i y-q}, \quad g_{ \pm}=\frac{\left(z \pm q^{2} / 2\right)^{2}-q^{2}}{2 q^{3}} \ln \frac{z+q \pm q^{2} / 2}{z-q \pm q^{2} / 2} .
$$

Коновские особенности определяются согласно формуле (3.16) из четырех уравнений: $q^{2} \pm 2 q \pm 2 z=0$. Эти уравнения при $y=0(\nu=0)$ определяют четыре коновские особенности, две из которых при $\omega \neq 0$ лежат в окрестности точки $q=2$ : $q_{1,2}=1+\sqrt{1 \pm 2 x}$, а две другие - в окрестности точки $q=-2: q_{3,4}=-1-\sqrt{1 \pm 2 x}$. При малых $x$ из этих формул имеем

$$
q_{1,2} \approx 2 \pm x \approx 2 \pm \frac{\omega}{k_{\mathrm{F}} v_{\mathrm{F}}}, \quad q_{3,4} \approx-2 \pm x \approx 2 \pm \frac{\omega}{k_{\mathrm{F}} v_{\mathrm{F}}}
$$




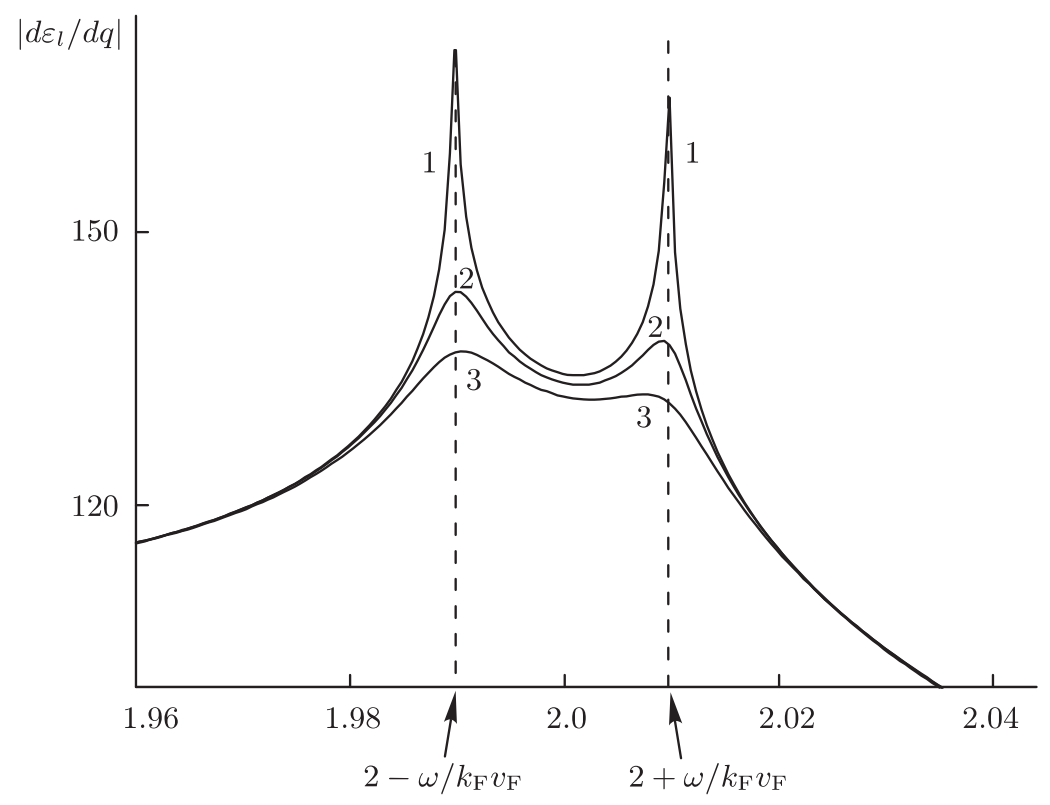

Рис. 3. Коновская особенность при $x_{\mathrm{p}}=10, x=0$.

В терминах размерного волнового числа Ферми коновские особенности определяются равенствами

$$
\begin{aligned}
& k_{1,2}=k_{\mathrm{F}}+\sqrt{k_{\mathrm{F}}^{2} \pm 2 \frac{k_{\mathrm{F}} \omega}{v_{\mathrm{F}}}} \approx 2 k_{\mathrm{F}} \pm \frac{\omega}{v_{\mathrm{F}}} \\
& k_{3,4}=-k_{\mathrm{F}}-\sqrt{k_{\mathrm{F}}^{2} \pm 2 \frac{k_{\mathrm{F}} \omega}{v_{\mathrm{F}}}} \approx-2 k_{\mathrm{F}} \pm \frac{\omega}{v_{\mathrm{F}}}
\end{aligned}
$$

Эти формулы можно переписать в виде

$$
k_{1,2}=\frac{m v_{\mathrm{F}}}{\hbar}\left[1+\sqrt{1 \pm \frac{2 \hbar \omega}{m v_{\mathrm{F}}^{2}}}\right], \quad k_{3,4}=\frac{m v_{\mathrm{F}}}{\hbar}\left[-1-\sqrt{1 \pm \frac{2 \hbar \omega}{m v_{\mathrm{F}}^{2}}}\right] .
$$

Таким образом, в бесстолкновительной плазме $(\nu=0)$ при $\omega \neq 0$ происходит расщепление коновских особенностей.

\section{4. СРАВНЕНИЕ С РЕЗУЛЬТАТОМ МЕРМИНА}

Мермином [24] было получено общее выражение для диэлектрической функции

$$
\varepsilon^{\mathrm{M}}(\omega, k)=1+\frac{(\omega+i \nu)\left[\varepsilon^{\circ}(\omega+i \nu, k)-1\right]}{\omega+i \nu \varepsilon^{\circ}(\omega+i \nu, k)-1 /\left(\varepsilon^{\circ}(0, k)-1\right)}
$$

на основе кинетического уравнения для одночастичной матрицы плотности $\rho$

$$
\frac{\partial \rho}{\partial t}+i[\mathcal{E}+V,+\rho]=\frac{\rho^{1 . e .}-\rho}{\tau}
$$


со временем релаксации $\tau$ и локально-равновесной матрицей плотности $\rho^{1 . e .}$, причем $\mathcal{E}$ - кинетическая энергия электронов, $V$ - самосогласованный потенциал, $[\cdot, \cdot]-$ коммутатор, $\rho^{\text {l.e. }}$ - локально-равновесная функция распределения электронов $\rho=$ $1 /\left(1+e^{\mathcal{E}-\mu-\delta \mu}\right), \mu-$ безразмерный (приведенный) химический потенциал.

Диэлектрическая функция вычисляется в импульсном представлении:

$$
f(\mathbf{p}, \mathbf{q}, t)=\left\langle\mathbf{p}+\frac{\mathbf{q}}{2}|\rho| \mathbf{p}-\frac{\mathbf{q}}{2}\right\rangle,
$$

где $|\mathbf{p}\rangle$ - собственное состояние импульса $\mathbf{p .}$ Для удобства можно преобразовать приведенную матрицу плотности к распределению Вигнера в фазовом пространстве:

$$
f(\mathbf{p}, \mathbf{R}, t)=\frac{1}{(2 \pi)^{3}} \int e^{i \mathbf{q} \mathbf{R}} f(\mathbf{p}, \mathbf{q}, t) d^{3} q,
$$

где $\mathbf{R}$ - пространственная координата.

В формуле (4.1) введено обозначение $\varepsilon^{\circ}(\omega, k)$ - так называемая диэлектрическая функция Линдхарда, т. е. диэлектрическая функция, полученная для бесстолкновительной плазмы и определяемая равенством (3.10) при $\nu=0$ :

$$
\varepsilon^{\circ}(\omega, k) \equiv \varepsilon_{1}(\omega, k)=1+\frac{3 \omega_{\mathrm{p}}^{2}}{2 k^{2} v_{\mathrm{F}}}\left[1-g\left(\omega_{+}, k\right)+g\left(\omega_{-}, k\right)\right] .
$$

Выражение $\varepsilon^{\circ}(\omega+i \nu, k)$ означает, что аргументы диэлектрической функции Линдхарда $\omega_{ \pm}$заменены формально на $\omega_{ \pm}+i \nu$, т. е.

$$
\varepsilon_{1}^{\circ}(\omega+i \nu, k)-1=\frac{3 \omega_{\mathrm{p}}^{2}}{2 k^{2} v_{\mathrm{F}}^{2}}\left[1-g\left(\omega_{+}+i \nu, k\right)+g\left(\omega_{-}+i \nu, k\right)\right]
$$

выражение $\varepsilon^{\circ}(0, k)$ означает, что в аргументах диэлектрической функции Линдхарда $\omega_{ \pm}$формально положили $\omega=0$, т. е.

$$
\varepsilon_{\mathrm{l}}^{\circ}(0, k)-1=\frac{3 \omega_{\mathrm{p}}^{2}}{2 k^{2} v_{\mathrm{F}}^{2}}\left[1-g\left(0_{+}, k\right)+g\left(0_{-}, k\right)\right] .
$$

Здесь

$$
g\left(0_{ \pm}, k\right)=\frac{m\left(\hbar k^{2} /\left(4 m^{2}\right)-v_{\mathrm{F}}^{2}\right)}{2 \hbar k v_{\mathrm{F}}} \ln \frac{ \pm \hbar k /(2 m)+v_{\mathrm{F}}}{ \pm \hbar k /(2 m)-v_{\mathrm{F}}} .
$$

Отсюда следует, что эти две функции отличаются лишь знаком: $g\left(0_{-}, k\right)=-g\left(0_{+}, k\right)$. Из формул (4.2) и (4.3) видно, что

$$
\frac{\varepsilon^{\circ}(\omega+i \nu, k)-1}{\varepsilon^{\circ}(0, k)-1}=\frac{1-g\left(\omega_{+}+i \nu\right)+g\left(\omega_{-}+i \nu\right)}{1-g\left(0_{+}, k\right)+g\left(0_{-}, k\right)} .
$$

С помощью соотношений (4.2)-(4.4) формула Мермина (4.1) в наших обозначениях записывается в виде

$$
\varepsilon^{\mathrm{M}}=1+\frac{3 \omega_{\mathrm{p}}^{2}}{2 k^{2} v_{\mathrm{F}}^{2}} \frac{(\omega+i \nu) g_{0}(k) G\left(\omega_{ \pm}, \nu, k\right)}{\omega g_{0}(k)+i \nu G\left(\omega_{ \pm}, \nu, k\right)},
$$


где

$$
g_{0}(k)=1-g\left(0_{+}, k\right)+g\left(0_{-}, k\right), \quad G\left(\omega_{ \pm}, \nu, k\right)=1-g\left(\omega_{+}+i \nu, k\right)+g\left(\omega_{-}+i \nu, k\right) .
$$

Из формул (3.10) и (4.5) видно, что при $\nu \rightarrow 0$ выведенная в работе формула и формула Мермина переходят в одну и ту же диэлектрическую функцию для квантовой бесстолкновительной плазмы - линдхардовскую диэлектрическую функцию (3.13).

В формуле Мермина (4.5) перейдем к тем же переменным (3.15), что и в формуле (3.16). В результате получаем следующее выражение для диэлектрической проницаемости:

$$
\varepsilon_{1}^{\mathrm{M}}(x, y, q)=1+\frac{3 x_{\mathrm{p}}^{2}}{2 q^{2}} \frac{(x+i y) g_{0}(q) G(z, q)}{x g_{0}(q)+i y G(z, q)} .
$$

Здесь введены следующие обозначения:

$$
\begin{gathered}
g_{0}(q)=1-g\left(0_{+}, k\right)+g\left(0_{-}, k\right)=1-2 g\left(0_{+}, k\right)=1-\frac{q^{2}-4}{4 q} \ln \frac{q+2}{q-2}, \\
G=1-g_{+}+g_{-}, \quad g_{ \pm}=\frac{\left(z \pm q^{2} / 2\right)^{2}-q^{2}}{2 q^{3}} \ln \frac{z+q \pm q^{2} / 2}{z-q \pm q^{2} / 2} .
\end{gathered}
$$

Формулы (4.6) и (3.16) представляют собой функции диэлектрического отклика квантовой вырожденной столкновительной плазмы, выведенные на основе кинетического уравнения, взятого соответственно в импульсном и координатном пространствах.

\section{5. ЗАКЛЮЧЕНИЕ}

Интересно отметить, что в низкочастотном пределе, т. е. при $\omega=0$, диэлектрическая функция Мермина не зависит от частоты столкновений $\nu$. В самом деле, полагая в формуле $(4.5) x=0(\omega=0)$, получаем

$$
\varepsilon_{1}^{\mathrm{M}}(0, y, q)=1+\frac{3 x_{\mathrm{p}}^{2}}{2}\left[1-\frac{q^{2}-4}{4 q} \ln \frac{q+2}{q-2}\right] .
$$

Из полученной в настоящей работе формулы (3.12) в низкочастотном пределе в безразмерных переменных (3.11) получается совсем другая формула:

$$
\begin{aligned}
\varepsilon_{1}(0, y, q) & =1+\frac{3 x_{\mathrm{p}}^{2}}{2}\left[1-\frac{i y}{2} \ln \frac{i y+1}{i y-1}\right]^{-1} \times \\
\times & {\left[1-\frac{(i y+q / 2)^{2}-1}{2 q} \ln \frac{i y+q / 2+1}{i y+q / 2-1}+\frac{(i y-q / 2)^{2}-1}{2 q} \ln \frac{i y-q / 2+1}{i y-q / 2-1}\right], }
\end{aligned}
$$

которая переходит в формулу (5.1) при $y=0$.

Отметим, что в низкочастотном статичном пределе $(\omega=0)$ мнимая часть диэлектрического отклика (3.12) равна нулю, и эта функция является действительнозначной и имеет следующий вид:

$$
\begin{aligned}
\varepsilon_{1}(0, y, q)=1 & +\frac{3 x_{\mathrm{p}}^{2}}{2(1-y \operatorname{arcctg} y)}\left[1+y\left(\operatorname{arcctg} \frac{1+q / 2}{y}+\right.\right. \\
& \left.\left.+\operatorname{arcctg} \frac{1-q / 2}{y}\right)-\frac{1+y^{2}-q^{2} / 2}{2 q} \ln \frac{y^{2}+(1-q / 2)^{2}}{y^{2}+(1+q / 2)^{2}}\right]
\end{aligned}
$$




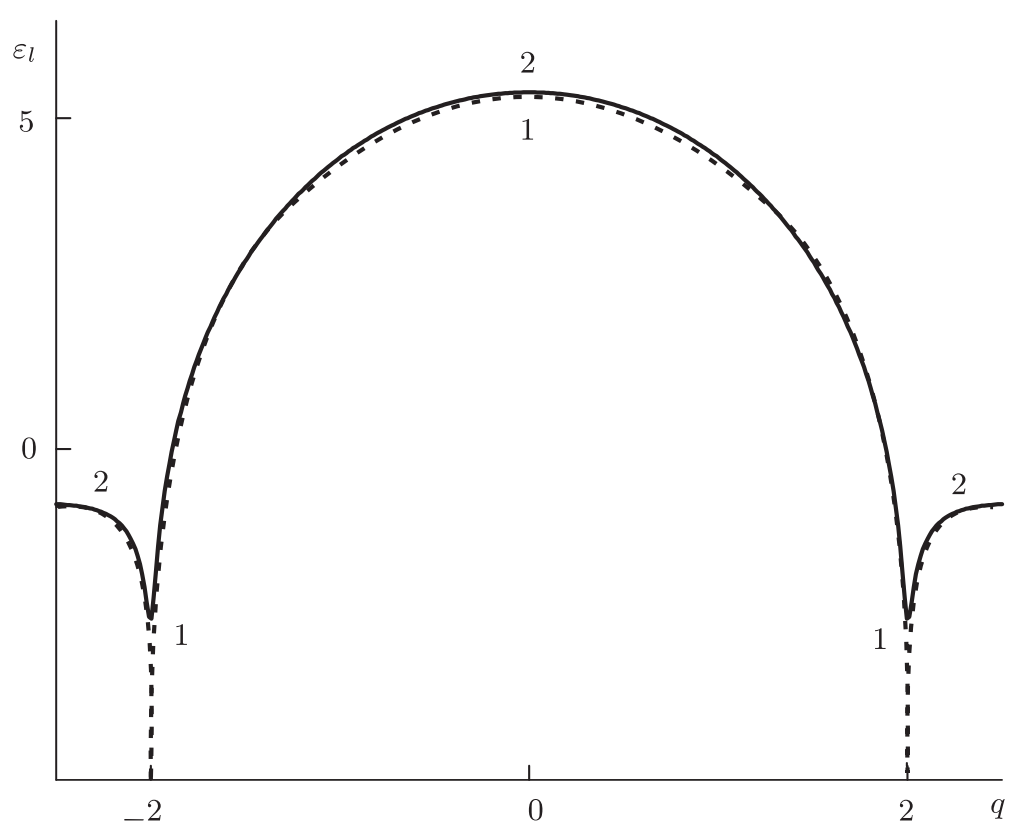

Рис. 4. Диэлектрический отклик для золота. Кривые отвечают двум значениям параметра $\varepsilon=\nu / \omega_{\mathrm{p}}: \varepsilon=10^{-5}$ (сплошная линия) и $\varepsilon=10^{-2}$ (штриховая линия).

Подчеркнем, что выражение (5.3) слабо зависит от частоты столкновений электронов. Для случая золота $\left(\omega_{\mathrm{p}}=1.37 \cdot 10^{16} \mathrm{c}^{-1}, v_{\mathrm{F}}=1.4 \cdot 10^{8} \mathrm{~cm} / \mathrm{c}, k_{\mathrm{F}}=8.2 \cdot 10^{8} \mathrm{~cm}^{-1}\right)$ на рис. 4 построены кривые диэлектрического отклика согласно формуле (5.3). Из рис. 4 видно, что эти кривые фактически совпадают, хотя эффективные частоты столкновений электронов различаются в 1000 раз. Отличие приведенных кривых проявляется лишь в окрестности коновских особенностей $(q= \pm 2)$. Таким образом, функция диэлектрического отклика в статическом пределе обнаруживает слабую зависимость от частоты столкновений электронов.

Итак, в настоящей работе получено аналитическое выражение для продольной диэлектрической проницаемости вырожденной электронной плазмы с учетом квантовых эффектов. Используется кинетическое БВВ-уравнение с интегралом столкновений в форме релаксационной $\tau$-модели в пространстве координат.

Показано, что в пределе, когда постоянная Планка стремится к нулю, полученное выражение переходит в классическую формулу для продольной диэлектрической проницаемости вырожденной плазмы.

Найдены статические пределы $(\omega \rightarrow 0)$ диэлектрической проницаемости как для бесстолкновительной, так и для столкновительной плазмы.

Отмечается расщепление коновских особенностей в бесстолкновительной плазме.

Приводится сравнение с классическим результатом Мермина для диэлектрической проницаемости. Отметим, что формула Мермина получена с использованием кинетического релаксационного уравнения в пространстве импульсов. Для бес- 
столкновительной плазмы как полученная в настоящей работе формула, так и формула Мермина переходят в одну и ту же формулу Линдхарда.

\section{Список литературы}

[1] П. К. Шукла, Б. Элиассон, УФН, 180:1 (2010), 55-82.

[2] B. Eliasson, P. K. Shukla, J. Plasma Phys., 76:1 (2010), 7-17, arXiv: 0911.4594.

[3] В. П. Силин, А. А. Рухадзе, Электромагнитные свойства плазмы и плазмоподобных сред, Госатомиздат, М., 1961.

[4] N. L. Tsintsadze, "Some New Aspects of Degenerate Quantum Plasma", New Frontiers in Advanced Plasma Physics, AIP Conf. Proc., 1306, eds. B. Eliasson, P. K. Shukla, AIP, Melville, N.Y., 2010, 75-88, arXiv: 1010.0073.

[5] Ю. Л. Климонтович, В. П. Силин, УФН, 70:2 (1960), 247-286.

[6] I. M. Gamba, M. P. Gualdani, C. Sparber, Kinetic and Related Modes, 2:1 (2009), 181-189.

[7] В. И. Татарский, УФН, 139:4 (1983), 587-619.

[8] G. Manfredi, "How to model quantum plasmas", Topics in Kinetic Theory (Toronto, Canada, March 24-26, 2004), Fields Inst. Commun., 46, eds. T. Passot, C. Sulem, P. L. Sulem, AMS, Providence, RI, 2005, 263-287, arXiv: quant-ph/0505004.

[9] D. Pines, J. Nucl. Energy C, 2:1 (1961), 5-17.

[10] A. Arnold, Transport Theory Statist. Phys., 30:4-6 (2001), 561-584.

[11] P. L. Bhatnagar, E. P. Gross, M. Krook, Phys. Rev., 94:3 (1954), 511-525.

[12] E. P. Wigner, Phys. Rev., 40:5 (1932), 749-759.

[13] M. Hillery, R. F. O'Connell, M. O. Scully, E. P. Wigner, Phys. Rep., 106:3 (1984), 121-167.

[14] Е. М. Лифшиц, Л. П. Питаевский, Теоретическая физика, т. 10: Физическая кинетика, Наука, М., 1979.

[15] Ф. Платцман, П. Вольф, Волны и взаимодействия в плазме твердого тела, Мир, М., 1975.

[16] J. Lindhard, Danske Vid. Selsk. Mat.-Fys. Medd., 28:8 (1954), 1-57.

[17] K. L. Kliewer, R. Fuchs, Phys. Rev., 181:2 (1969), 552-558.

[18] R. Fuchs, K. L. Kliewer, Phys. Rev., 185:3 (1969), 905-913.

[19] R. Fuchs, K. L. Kliewer, Phys. Rev. B, 3:7 (1971), 2270-2278.

[20] W. Kohn, S. H. Vosko, Phys. Rev., 119:3 (1960), 912-918.

[21] W. Kohn, L. J. Sham, Phys. Rev., 137:6A (1965), A1697-A1705.

[22] У. Харрисон, Теория твердого тела, Мир, М., 1972.

[23] П. А. Эминов, ЖЭТФ, 135:5 (2009), 1029-1036.

[24] N. D. Mermin, Phys. Rev. B, 1:5 (1970), 2362-2363.

[25] J. Kroha, J. Non-crystalline Solids, 250-252 (1999), 865-868, arXiv: cond-mat/9810068.

[26] К. П. Гуров, Основания кинетической теории. Метод Н.Н. Боголюбова, Наука, М., 1966.

[27] M. Opher, G. J. Morales, J. N. Leboeuf, Phys. Rev. E, 66:1 (2002), 016407, 10 pp.

Поступила в редакцию 14.11.2010, после доработки 17.03.2011 